\title{
Ovarian mature cystic teratoma with fistula formation into the rectum: a case report
}

\author{
Yuichiro Kizaki, Tomonori Nagai ${ }^{*}$ (D) Ken Ohara, Yosuke Gomi, Taichi Akahori, Yoshihisa Ono, \\ Shigetaka Matsunaga, Yasushi Takai, Masahiro Saito, Kazunori Baba and Hiroyuki Seki
}

\begin{abstract}
Background: While ovarian mature cystic teratomas are benign ovarian germ-cell tumors and the most common type of all ovarian tumors, the formation of fistulas into surrounding organs such as the bladder and the intestinal tract is extremely rare. This report documents a case of ovarian mature cystic teratoma with a rectal fistula, thought to be caused by local inflammation.

Case description: A pelvic mass was diagnosed as an ovarian mature cystic teratoma of approximately $10 \mathrm{~cm}$ in diameter on transvaginal ultrasound and magnetic resonance examinations. Endoscopic examination of the lower gastrointestinal tract to investigate diarrhea revealed an ulcerative lesion with hair in the rectal wall adjacent to the ovarian cyst, and formation of a fistula from the ovarian teratoma into the rectum was suspected. Laparotomy revealed extensive inflammatory adhesions between a left ovarian tumor and the rectum. Left salpingo-oophorectomy and upper anterior resection of the rectum were performed. The final pathological diagnosis was ovarian mature cystic teratoma with no malignant findings, together with severe rectal inflammation and fistula formation with no structural disorders such as diverticulitis of the colon or malignant signs.
\end{abstract}

Discussion: The formation of fistulas and invasion into the neighboring organs are extremely rare complications for ovarian mature cystic teratomas. The invasion of malignant cells into neighboring organs due to malignant transformation of the tumor is reported as the cause of fistula formation into the neighboring organs. A review of 17 cases including the present case revealed that fistula formation due to malignant transformation comprised only 4 cases $(23.5 \%)$, with inflammation as the actual cause in the majority of cases (13 cases, $76.5 \%$ ).

Conclusion: Although malignancy is the first consideration when fistula formation is observed between ovarian tumors and surrounding organs, in mature cystic teratoma, local inflammation is more likely than malignant transformation.

Keywords: Diarrhea, Fistula, Inflammation, Ovarian neoplasms, Teratoma

\section{Background}

Ovarian mature cystic teratomas are benign ovarian germ-cell tumors and the most common type of all ovarian tumors, comprising 10-25\% of cases (Tandon et al. 2010). The most common known complication is torsion

${ }^{*}$ Correspondence: tomono@saitama-med.ac.jp

Department of Obstetrics and Gynecology, Saitama Medical Center,

Saitama Medical University, 1981 Kamoda, Kawagoe-shi, Saitama, Japan of the tumor, reportedly observed in approximately $16 \%$ of cases (Tandon et al. 2010; Park et al. 2008). Meanwhile, formation of fistulas into neighboring organs is an extremely rare complication, occurring in less than $1 \%$ of cases (Tandon et al. 2010; von-Walter 2012) and seldom reported. In the present case, endoscopy of the lower gastrointestinal tract performed to investigate refractory diarrhea resulted in the incidental discovery of a rectal fistula originating from an ovarian mature cystic teratoma. 


\section{Case description}

The patient in this case was a 43-year-old woman. She had no significant past medical history and no familial history such as cancer and inflammatory bowel disease. She was examined by her local physician because of flulike symptoms, fever, and diarrhea. Abdominal ultrasound examination incidentally revealed a pelvic mass, and she was referred to our institution. Transvaginal ultrasound examination revealed an $11 \mathrm{~cm}$ pelvic mass (Fig. 1). Inside the mass, suspected hair was observed in

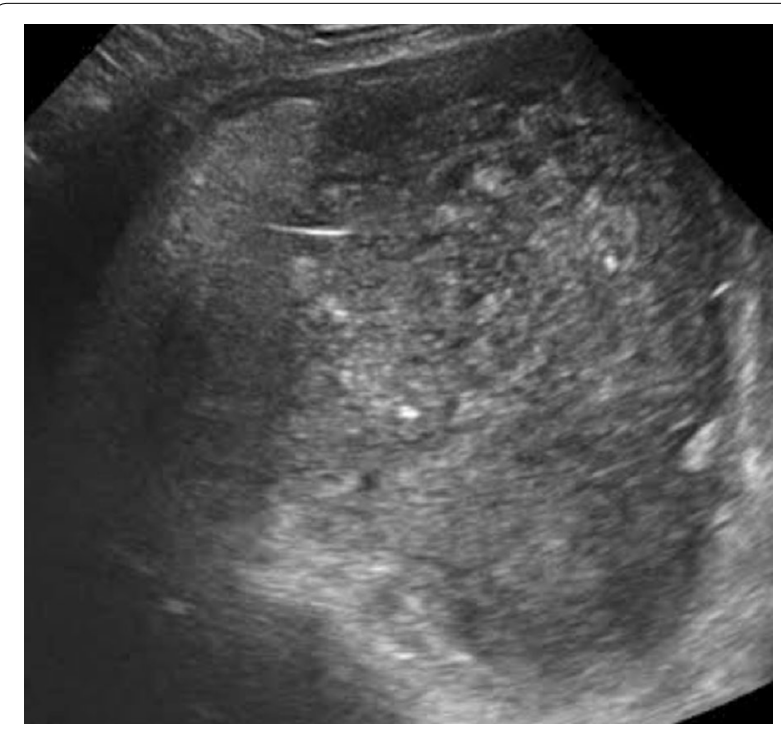

Fig. 1 Transvaginal ultrasound image. A pelvic mass is seen of approximately $11 \mathrm{~cm}$ in diameter and is suspected to contain hair because of the presence of hyperechoic lines the form of hyperechoic lines, leading to suspicion of an ovarian mature cystic teratoma. Blood and biochemical tests did not reveal blood-count or other abnormalities. Serum levels of tumor markers including carcinoembryonic antigen (CEA; $1.0 \mathrm{ng} / \mathrm{mL}$ ), carbohydrate antigen 125 (CA125; $59 \mathrm{U} / \mathrm{mL}$ ), and carbohydrate antigen 19-9 (CA19-9; $9 \mathrm{U} / \mathrm{mL}$ ) were within normal range, but that for squamous cell carcinoma antigen (SCC; $2.0 \mathrm{ng} / \mathrm{mL}$ ) was elevated. Abdominal contrast-enhanced computed tomography $(\mathrm{CT})$ revealed an $11 \mathrm{~cm}$ cystic tumor in the pelvis, containing lipids and a fat-fluid level. No distinct abnormalities were observed in other organs.

The fat-fluid level inside the tumor was also observed on pelvic contrast-enhanced magnetic resonance imaging (MRI), with high signal intensity on the abdominal side in both T1- and T2-weighted images and low signal intensity on fat-suppression images, suggesting ovarian mature cystic teratoma (Fig. 2a, b). No solid areas with contrast enhancement to suggest malignancy were observed inside the tumor. From the imaging findings, an ovarian mature cystic teratoma was suspected and the decision was made to perform laparoscopic surgery.

The patient also consulted the local medical gastroenterology clinic because of ongoing symptoms of diarrhea ranging in color from white to bright green. Endoscopic examination of the lower gastrointestinal tract revealed hair and a submucosal tumor-like protrusion with redness at its apex, situated approximately $12 \mathrm{~cm}$ adoral to the anal orifice (Fig. 3). No signs of diverticulitis were observed in the surrounding area. As this lesion site was adjacent to the ovarian tumor, and hair was present at the lesion site, formation of a fistula from the ovarian mature cystic teratoma into the rectum was suspected.
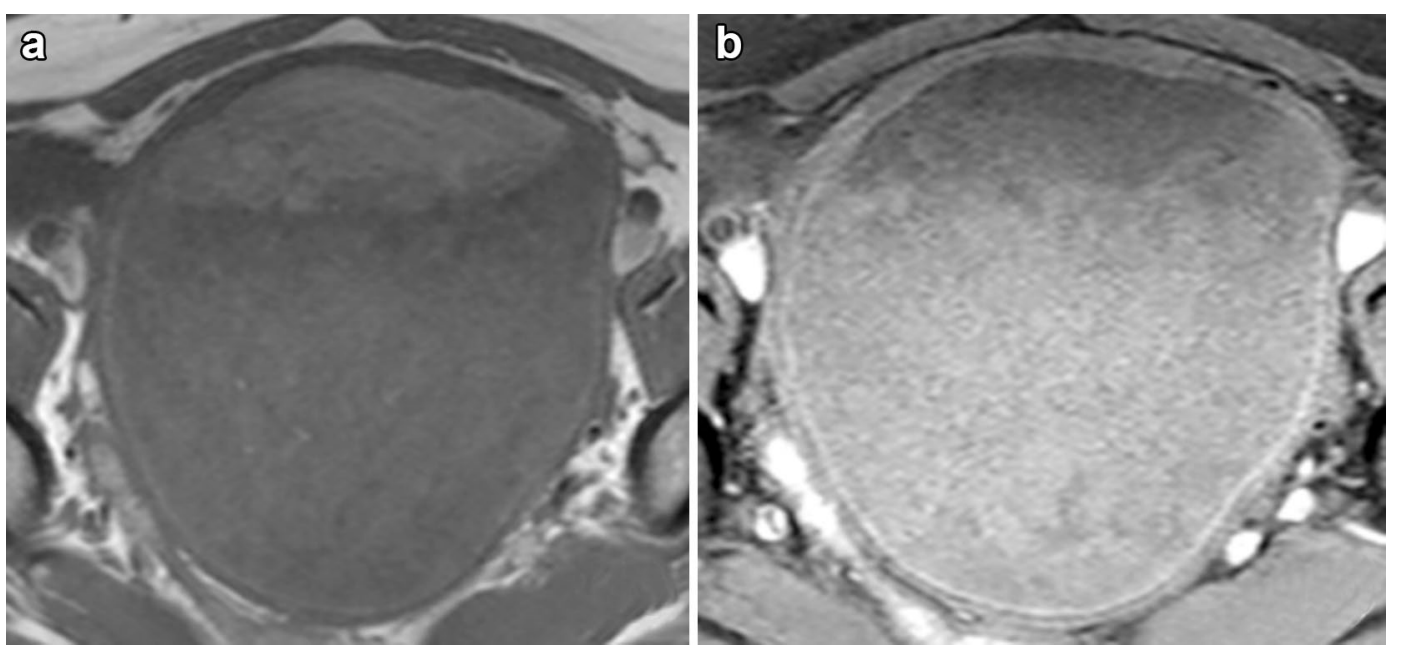

Fig. 2 Magnetic resonance images. a A T1-weighted image shows a cystic tumor containing a fat-fluid level. b A fat-suppression image reveals fat suppression on the abdominal side of the niveau 


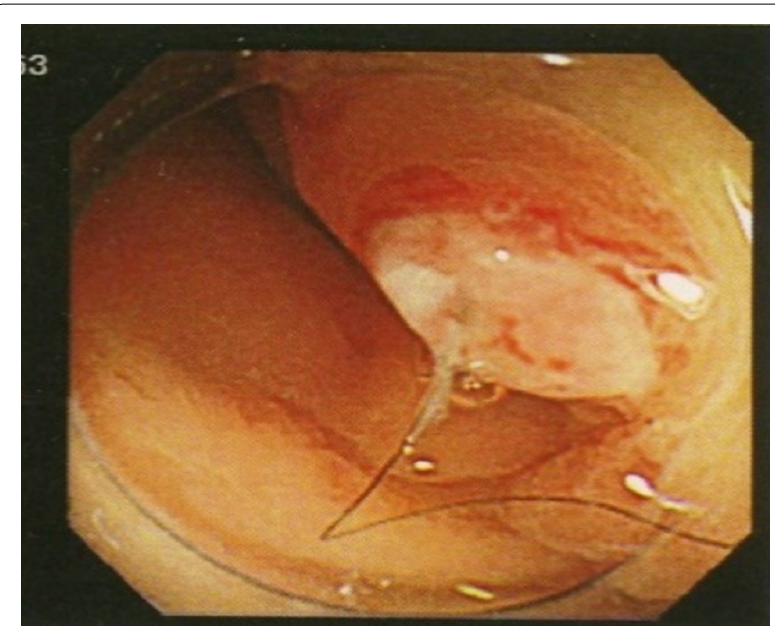

Fig. 3 Lower gastrointestinal tract endoscopy image. Hair and a submucosal tumor-like protrusion with redness at its apex are seen

As rectal invasion by a benign mature cystic teratoma is very unlikely, the possibility of malignant transformation of the mature cystic teratoma was considered, and the decision was made to perform salpingo-oophorectomy of the diseased side via laparotomy rather than laparoscopic surgery.

Surgical findings were as follows. The left ovary was enlarged to approximately $10 \mathrm{~cm}$ in diameter and occupied most of the pelvic cavity. The ovarian tumor had a smooth surface, and part of the dorsal side was closely adhered to the rectum (Fig. 4a). The tumor was carefully separated from the rectum, and drainage of the tumor contents from the separation site revealed bright-green pus with a strong odor. After completion of the left salpingo-oophorectomy, the resected tissue was submitted for frozen-section pathological diagnosis during surgery. The resulting diagnosis was ovarian mature cystic teratoma accompanied by inflammatory granulation, with no malignant findings. In addition, a fistula of approximately $1 \mathrm{~cm}$ in diameter was observed in the anterior rectal wall at the site of separation from the tumor (Fig. 4b). Based on the result of frozen-section, it was possible to perform partial resection of rectal wall as minimal surgery, but necessity of histological examination of the tissue surrounding the rectal fistula was considered, and upper anterior resection of the rectum without temporary colostomy was performed at same time following the left salpingo-oophorectomy. Postoperative progress was satisfactory, and the patient was discharged on the 9th day after surgery. The final pathological diagnosis was identical to the pathological diagnosis during surgery, confirming a benign left-sided ovarian mature cystic teratoma. Findings of abundant inflammatory cell invasion in the cyst wall and hair-shaft tissue were consistent with a mature cystic teratoma (Fig. 5a). Further, although inflammatory cell invasion and hair-shaft tissue were observed in the rectal serosa, no structural disorders such as diverticulitis of the colon or signs of malignancy were observed (Fig. 5b).

\section{Discussion}

Ovarian mature cystic teratomas are the most common ovarian tumor, comprising 10-25\% of all cases (Tandon et al. 2010). Multiple complications are known, of which the most common is torsion (16\% of tumors). Other reported complications include rupture (1-4\%), malignant transformation (1-2\%), and infection (1\%) (Park et al. 2008). Meanwhile, the formation of fistulas and invasion into the neighboring organs are extremely rare
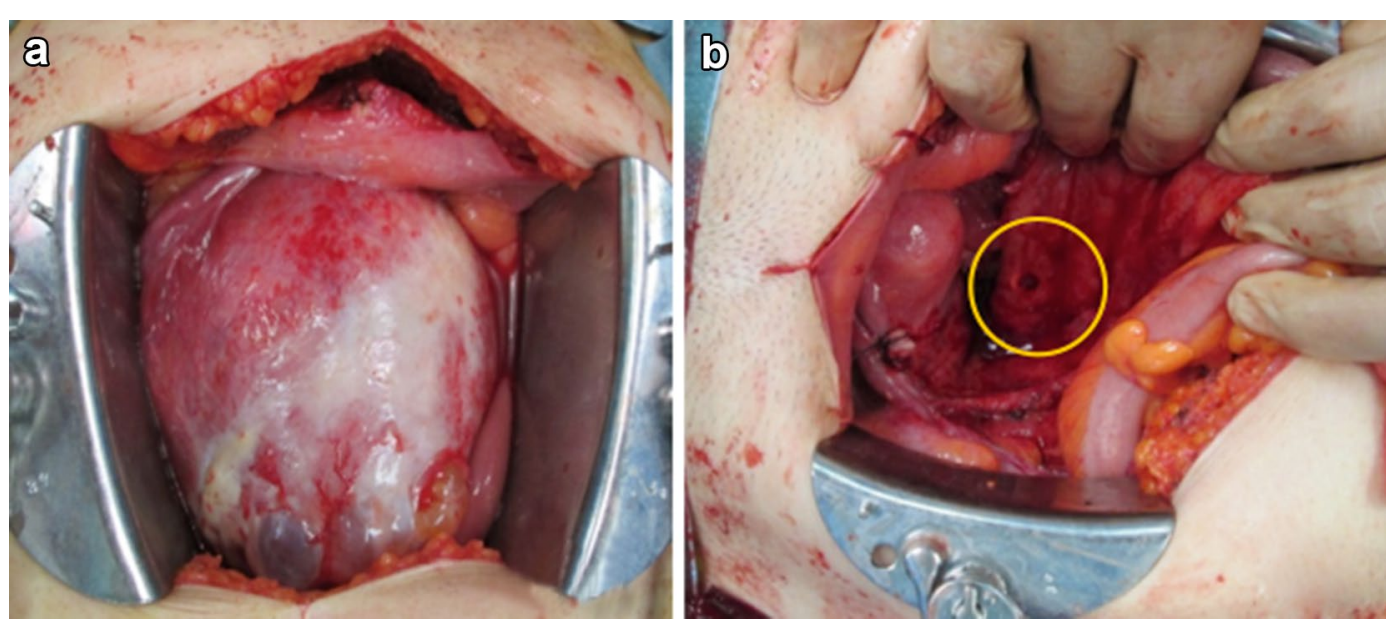

Fig. 4 Surgical images. a An ovarian tumor with a smooth surface occupies the pelvic cavity. b After left salpingo-oophorectomy, a fistula of approximately $1 \mathrm{~cm}$ in diameter is seen on the surface of the rectum at the site of adhesion 

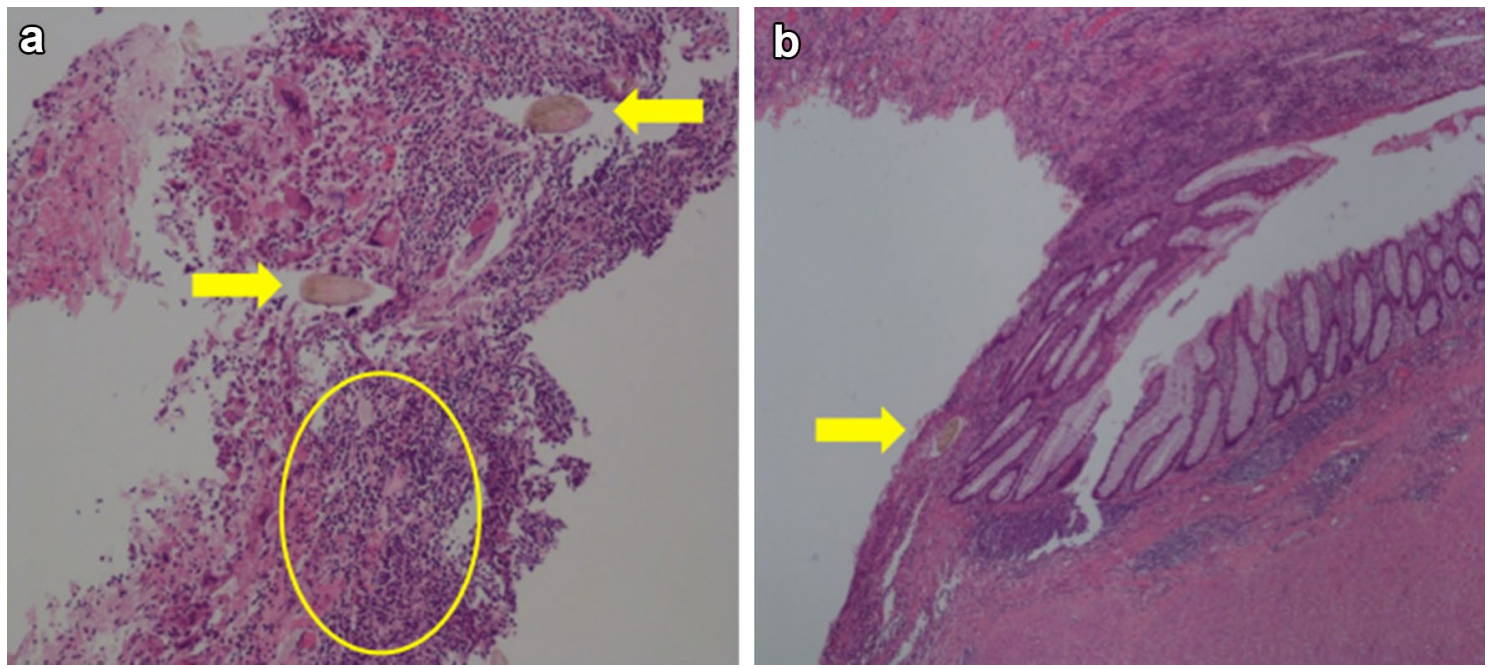

Fig. 5 Histopathological images. a A histological section of the mature cystic teratoma reveals abundant inflammatory cell invasion (circled section) and hair-shaft tissue (arrows). b A section of the rectal-fistula site shows hair-shaft tissue in the serous membrane of the rectum (arrow)

complications for this tumor, with frequency said to be less than $1 \%$ (Tandon et al. 2010; von-Walter 2012).

The invasion of malignant cells into neighboring organs due to malignant transformation of the tumor is reported as the cause of fistula formation into the neighboring organs (Mitui et al. 1983; Okada et al. 2005; Song and Conner 2012; Gooneratne et al. 2015), but malignant transformation is not a necessary condition for the formation of fistulas (Kim et al. 1999).

In this instance, we performed surgery in consideration of the possible presence of a malignant tumor as the cause of fistula formation. Pathological diagnosis performed during surgery identified the tumor as a benign ovarian mature cystic teratoma. Histological examination of the area around the rectal fistula was deemed necessary to rule out the possibility of a malignant tumor, and an upper anterior resection of the rectum was performed. However, no signs of malignancy were found, and it was concluded that the fistula formation was due to inflammation. A retrospective review would suggest that the surgery conducted in this case (upper anterior resection of the rectum) was over-treatment, and trimming and restoration of the fistula site might have been sufficient. However, we believed that malignancy together with the invasion of malignant cells into neighboring organs was a necessary condition for the formation of fistulas.

Considering malignancy as a prerequisite for fistula formation into neighboring organs from ovarian mature cystic teratomas, we conducted a literature search and found 16 reported cases during the period from 1938 to 2015. A short review of these cases is shown in Table 1 together with the case we experienced. Of the 17 cases, fistula formation due to malignant transformation comprised only 4 cases $(23.5 \%)$, with inflammation as the actual cause in the majority of cases (13 cases, $76.5 \%$ ). However, one could still argue with a $23.5 \%$ chance of malignancy that we performed an appropriate surgery. We may have been able to modify our policy once we received the pathological diagnosis during the surgery, but there is a risk of under-diagnosis.

Shiels et al. (1986) consider the following steps to be involved in the pathogenesis of fistula formation: (1) rupture or perforation of the cyst, (2) small leakage from the cyst causing dense adhesion between the cyst and the surrounding organs, (3) circulatory disturbance of the walls of these structures due to the resultant necrosis and inflammation, and (4) formation of fistulas in the walls of these structures. In addition, they have mentioned that rupture or perforation of the cyst is likely caused by circulatory disorders or infection due to factors such as partial torsion, malignant transformation, and mechanical forces.

Fistula formation was observed in 20 sites in 17 patients, listed by organ as the urinary bladder ( 5 cases, $25.0 \%$ ), small intestine (5 cases, $25.0 \%$ ), sigmoid colon (4 cases, $20.0 \%$ ), rectum (4 cases, $20.0 \%$ ), and transverse colon ( 2 cases, $5.0 \%$ ). While Kim et al. (1999) have reported that fistulas are most likely to form in the urinary bladder, we consider their formation equally likely in any organ neighboring the tumor, or more likely to form in the lower gastrointestinal tract. Further, the symptoms presented will depend on the organ into which a fistula has formed.

Of the 13 cases in which inflammation was the cause, including the case we experienced, extended surgery 
Table 1 Reported cases of ovarian teratoma complicated with fistula formation

\begin{tabular}{|c|c|c|c|c|c|}
\hline $\begin{array}{l}\text { Author (date of publica- } \\
\text { tion) }\end{array}$ & Age & Symptoms & Involved organ & Surgical method & Cause of fistula formation \\
\hline Robinson (1938) & 64 & Cystitis, dysuria & Bladder & USO, PC & Inflammation \\
\hline Mitui et al. (1983) & 72 & Diarrhea containing hair & $\begin{array}{l}\text { Sigmoid colon, small } \\
\text { intestine }\end{array}$ & RS, colostomy, PRSI & Malignant transformation \\
\hline Shiels et al. (1986) & 21 & Nausea, dyspareunia & Sigmoid colon & $\begin{array}{l}\text { USO, fistulectomy, repair of } \\
\text { the bowel defect }\end{array}$ & Inflammation \\
\hline Ulstein et al. (1987) & 30 & Bladder stone & Bladder & USO, PC & Inflammation \\
\hline Landmann et al. (1988) & 22 & Rectal bleeding & Rectum & USO, LAR & Inflammation \\
\hline Suzuki et al. (1999) & 64 & Microscopic hematuria & Bladder, small intestine & $\begin{array}{l}\text { TH, BSO, appendectomy, } \\
\text { PRSI, PC }\end{array}$ & Inflammation \\
\hline Tabata et al. (2004) & 20 & Pyuria & Bladder & USO,PC & Inflammation \\
\hline Okada et al. (2005) & 54 & $\begin{array}{l}\text { Abdominal pain, watery } \\
\text { diarrhea }\end{array}$ & Small intestine & TH, BSO, PRSI, POM & Malignant transformation \\
\hline Cebesoy et al. (2009) & 30 & $\begin{array}{l}\text { Abdominal pain, purulent } \\
\text { diarrhea }\end{array}$ & Rectum & USO, LAR & Inflammation \\
\hline Tandon et al. (2010) & 30 & Pyuria, dysuria & Bladder & USO,PC & Inflammation \\
\hline Rajaganeshan et al. (2011) & 44 & $\begin{array}{l}\text { Weight loss, loose stools, } \\
\text { rectal bleeding }\end{array}$ & Rectum & $\begin{array}{l}\text { USO, repair of the bowel } \\
\text { defect }\end{array}$ & Inflammation \\
\hline Salame et al. (2011) & 38 & None & Sigmoid colon & RS & Inflammation \\
\hline von-Walter et al. (2012) & 25 & Small bowel obstruction & $\begin{array}{l}\text { Small intestine, transverse } \\
\text { colon }\end{array}$ & $\begin{array}{l}\text { USO, PRSI, repair of the } \\
\text { bowel defect }\end{array}$ & Inflammation \\
\hline Song et al. (2012) & 73 & $\begin{array}{l}\text { Abdominal pain, constipa- } \\
\text { tion }\end{array}$ & Small intestine & USO, PRSI & Malignant transformation \\
\hline Conway et al. (2012) & 26 & Abdominal pain, nausea & Transverse colon & Repair of the bowel defect & Inflammation \\
\hline Gooneratne et al. (2015) & 63 & $\begin{array}{l}\text { Abdominal distention, } \\
\text { bloody diarrhea }\end{array}$ & Sigmoid colon & Exploratory laparotomy & Malignant transformation \\
\hline Our case & 43 & Diarrhea & Rectum & USO, HAR & Inflammation \\
\hline
\end{tabular}

USO unilateral salpingo-oophorectomy, BSO bilateral salpingo-oophorectomy, $T H$ total hysterectomy, $P C$ partial cystectomy, $R S$ recto-sigmoidectomy, $P R S /$ partial resection of small intestine, $L A R$ lower anterior resection, HAR high anterior resection, POM partial omentectomy

appears to have been performed because of the possibility of malignancy in at least 5 cases (38.5\%). We too performed extended surgery in consideration of the possibility of a malignant tumor, but with the knowledge that inflammation is an extremely common cause of fistula formation from ovarian mature cystic teratomas, more limited surgery might have been possible, and we must reflect upon this point.

\section{Conclusions}

Formation of fistulas into neighboring organs is an extremely rare complication of ovarian mature cystic teratomas. In the present case, we experienced the fistula formation into the rectum, and concerned about the possibility of malignancy, nevertheless no malignancy was observed. While opportunities to encounter such a case in clinical practice seldom arise, if a case is encountered or this complication is suspected, treating doctors must remember that the presence of malignant transformation is not a necessary condition for formation, and in fact inflammation is the cause in most cases. While surgical intervention is necessary when the formation of fistulas is observed, it should be performed subject to a thorough assessment of the possible causes and with due attention to the avoidance of over-treatment.

\begin{abstract}
Authors' contributions
YK was a major contributor in writing the manuscript. TN contributed to the study concept and design and was involved in drafting and revising the manuscript. KO, YG, TA and SM participated in the actual treatment of the patient. YO, MS and YT were involved in revising the manuscript. KB carried out the ultrasonographic measurements. HS supervised the study, critiqued the study design, edited and commented on the manuscript. All authors read and approved the final manuscript.
\end{abstract}

\section{Acknowledgements}

The authors thank all the doctors in our department for their assistance during this study.

\section{Competing interests}

The authors declare that they have no competing interests.

\section{Consent}

Written informed consent was obtained from the patient for the publication of this report and any accompanying images.

Received: 24 February 2016 Accepted: 28 September 2016

Published online: 03 October 2016 


\section{References}

Cebesoy FB, Baskonus I, Mete A, Kutlar I, Aybasti N (2009) Benign ovarian dermoid cyst complicated with rectal fistula formation: an unusual case. Arch Gynecol Obstet 279:179-181

Conway AZ, Hanna T (2012) A dermoid cyst fistulating with the transverse colon. Ann R Coll Surg Engl 94:e230-e231

Gooneratne AT, James AO, Gupta J, Abdulaal Y (2015) Squamous cell carcinoma arising in a mature cystic teratoma invading the sigmoid colon: a rare presentation. BMJ Case Rep. doi:10.1136/bcr-2014-208472

Kim SJ, Kimoto Y, Nakamura H, Taguchi T, Tanji Y, Shiba E, Takai S (1999) Ovarian carcinoma with fistula formation to the sigmoid colon and ileum: report of a case. Surg Today 29:449-452

Landmann DD, Lewis RW (1988) Benign cystic ovarian teratoma with colorec tal involvement. Report of a case and review of the literature. Dis Colon Rectum 31:808-813

Mitui AH, Fujita R, Sugata F, Kienebuchi M, Suzuki K, Sagawa F (1983) A case of ovarian dermoid cyst with malignant transformation perforated into the rectosigmoid colon and small intestine. Endoscopy 15:331-333

Okada S, Ohaki Y, Inoue K, Nakajo H, Kawamata H, Kumazaki T (2005) A case of dermoid cyst of the ovary with malignant transformation complicated with small intestinal fistula formation. Radiat Med 23:443-446

Park SB, Kim JK, Kim KR, Cho KS (2008) Imaging findings of complications and unusual manifestations of ovarian teratomas. Radiographics 28:969-983

Rajaganeshan R, Wang H, Abouleid A, Scott G, Selvasekar CR (2011) Conservative surgery in the management of a benign ovarian cystic teratoma presenting as a rectal mass: a case report. Ann R Coll Surg Engl 93:e46-e48
Robinson RH (1938) Ovarian Teratoma, invading the bladder. Proc R Soc Med 31:1076-1077

Salame G, Sherer DM, Shah T, Serur E, Dalal S, Dalloul M, Abulafia O (2011) Mature cystic teratoma of the sigmoid colon. Ultrasound Obstet Gynecol 37:739-740

Shiels WE, Dueno F, Hernandez E (1986) Ovarian dermoid cyst complicated by an entero-ovarian fistula. Radiology 160:443-444

Song W, Conner M (2012) Squamous cell carcinoma arising within a mature cystic teratoma with invasion into the adjacent small intestine: a case report. Int J Gynecol Pathol 31:272-275

Suzuki M, Fukasawa M, Hara T (1999) Dermoid cyst with thyroid follicle perforated into bladder and ileum: a case report. Nippon Hinyokika Gakkai Zasshi 90:586-589

Tabata K, Nagakura K, Hirano I, Baba S (2004) A case of fistula between the urinary bladder and an ovarian dermoid cyst. Hinyokika Kiyo 50:219-221

Tandon A, Gulleria K, Gupta S, Goel S, Bhargava SK, Vaid NB (2010) Mature ovarian dermoid cyst invading the urinary bladder. Ultrasound Obstet Gynecol 35:751-753

Ulstein M, Baydia JL, Pradhan U (1987) Fistula between the urinary bladder and an ovarian dermoid tumor. Acta Obstet Gynecol Scand 66:723-724

von Walter AR, Nelken RS (2012) Benign cystic ovarian teratoma with a fistula into the small and large bowel. Obstet Gynecol 119(2 Pt 2):434-436

\section{Submit your manuscript to a SpringerOpen ${ }^{\circ}$ journal and benefit from:}

- Convenient online submission

- Rigorous peer review

- Immediate publication on acceptance

- Open access: articles freely available online

- High visibility within the field

- Retaining the copyright to your article

Submit your next manuscript at $\boldsymbol{\nabla}$ springeropen.com 